\title{
Low Energy Communication Protocol for Implantable Wireless Body Sensor Networks
}

\author{
Maryam Elazhari, Ahmed Toumanari, Rachid Latif, Nadya El moussaid \\ ESSI, National School of applied Sciences, Ibnou zohr university, Agadir Morocco
}

\begin{abstract}
IWBSN (Implantable Wireless Body Sensor Network) becomes nowadays an important area of research in computer science and healthcare application industries for improving the quality of life. Communication with implanted medical devices is considered as a key to effective diagnosis and therapy. These biosensors are implanted inside the patient body to measure body temperature and blood pressure, respiration rate, blood pressure and other physiological parameters. The purpose of this paper is to provide an enhanced version of TBCD communication protocol (Time Based Coded Data) for implantable sensor networks in order to guarantee an ultra-low energy consumption in the very tiny battery of the biosensors, and hence increasing the network lifetime for longer periods of time.
\end{abstract}

\section{Keywords}

Biomedical, low-power communication, wireless, body sensor networks, implantable sensors, healthcare applications, biosensors.

\section{INTRODUCTION}

Implantable biosensors are an important class of biosensors based on their ability to continuously measure metabolite levels, without the need for patient intervention and regardless of the patient's physiological state (sleep, rest, etc.). For instance, implantable biosensors represent a highly desirable proposition for diabetes management which currently relies on data obtained by using test strips blood from finger pricking, a procedure which is not only painful, but also is incapable of reflecting the overall direction, trends, and patterns associated with daily habits [1-4]. The development of implantable sensors offers BSN one of its most exciting components. The European Commission project "Healthy Aims" has been focused on specific sensor applications, namely for hearing aids (cochlear implant), vision aids (retinal implant), detecting raised orbital pressure (glaucoma sensor), and intracranial pressure sensing (implantable pressure sensor).Other implantable devices include Medtronic's "Reveal Insertables Loop Recorder", which is a fully implantable cardiac monitor used to record the heart's rate and rhythm during instances of unexplained fainting, dizziness, or palpitations. The device provides the clinician with an ECG that can be used to identify or rule out an abnormal heart rhythm as the cause of these symptoms. CardioMEMS is a company that produces an implantable pressure sensor, which has been developed at Georgia Institute of Technology that can take pressure readings following implantation into an aneurism sac at the time of endovascular repair. This implanted sensor then provides a means of monitoring the status of the repair during the years following. Finally, Given Technologies has developed an endoscopy capsule that transmits images of the small bowel as it travels through the gastrointestinal tract. Fig 1 below shows some of the implantable body cavity sensing technologies that may ultimately form part of an implantable wireless BSN.
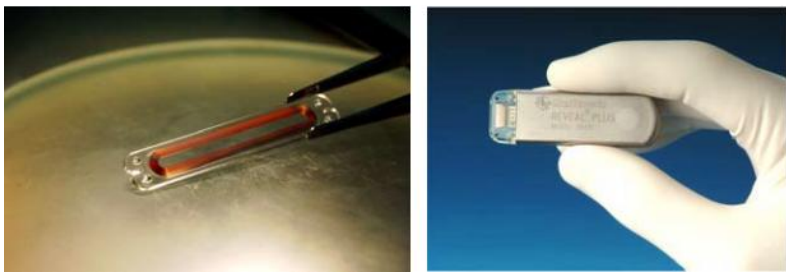

Fig 1: (Left) The CardioMEMS Endosure wireless aneurism pressure- sensing device. (Right) Medtronic "Reveal Insertable Loop Recorder".

Other implantable devices currently used in clinical practice include implantable drug delivery systems for chronic pain, sacral nerve stimulators for anal incontinence, and high frequency brain (thalamic) stimulation for neurological conditions such as Parkinson's disease and refractory epilepsy Fig 2 shows a range of these implantable devices currently in clinical use [5]-[7].
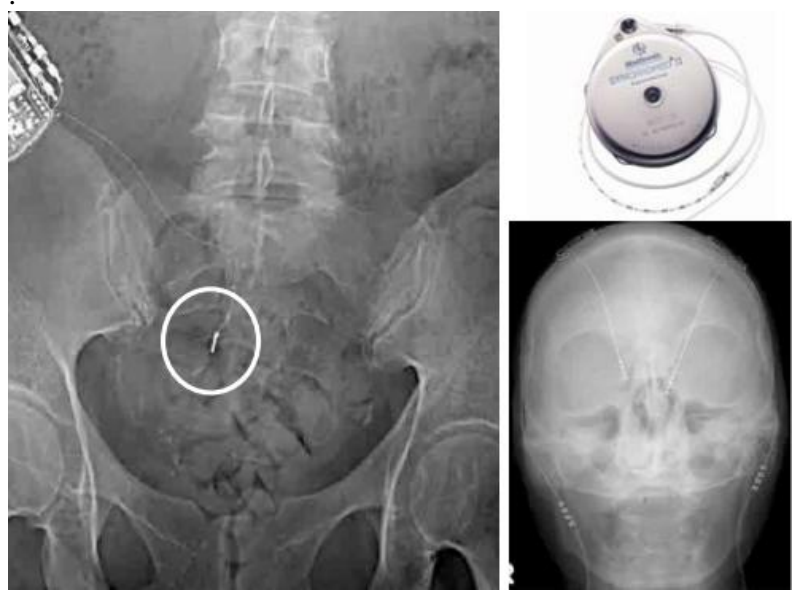

Fig 2: (Left) An example of an implantable device (sacral nerve stimulator) in current clinical use. (Bottom right) Deep brain stimulators, (courtesy of the Radiological Society of North America). (Above right) Implantable Synchromed II drug delivery system for chronic pain (courtesy of Medtronic Inc.). 


\section{REVIEW OF TBCD ROUTING PROTOCOL}

When considering wireless transmission around and on the body, important issues are radiation absorption and heating effects on the human body. To reduce tissue heating the radio's transmission power can be limited or traffic control algorithms can be used. In [8] rate control is used to reduce the bioeffects in a single-hop network. Another possibility is a protocol that balances the communication over the sensor nodes. An example is the Thermal Aware Routing Algorithm (TARA) that routes data away from high temperature areas (hot spots) [9]. Packets are withdrawn from heated zones and rerouted through alternate paths. TARA suffers from low network lifetime, a high ratio of dropped packets and does not take reliability into account. An improvement of TARA is Least Temperature Routing (LTR) and Adaptive Least Temperature Routing (ALTR) [10] that reduces unnecessary hops and loops by maintaining a list in the packet with the recently visited nodes. ALTR switches to shortest hop routing when a predetermined number of hops is reached in order to lower the energy consumption. A smarter combination of LTR and shortest path routing is Least Total Route Temperature (LTRT) [11]. The node temperatures are converted into graph weights and minimum temperature routes are obtained. A better energy efficiency and a lower temperature rise is obtained, but the protocol has as main disadvantage that a node needs to know the temperature of all nodes in the network. Daniel Garrison presents in [12] a model of data reporting that balances power consumption with body temperature thresholds. The main disadvantage of the algorithm is that a cluster head drains its energy rapidly since no rotation role of cluster head is taking place, moreover, sensor nodes will have to send data directly to the sink node during the cooling down period, thus minimizing network lifetime.TBCD communication protocol is an ultra low energy communication protocol for IWBSNs There is two special counters in this protocol required in the entire network in each sensor node and the base station. These counters play the main role of the concept in the algorithm. These two counters are called ID-Counter and Data_Counter. The ID_Counter is for identifying the sensor ID, and the Data_Counter is to search for the corresponding data to be sampled by the sensor in the node. These two counters in the design are concatenated to each other so that the lower order is Data_Counter and the higher order is ID_Counter (see Figure 3). Whenever the unsigned value of the Data_Counter in any sensor node or in the BS overflows (which is supposed to happen for all nodes at the same time because the synchronization has already been established with respect to the wireless delay), then at this moment its associated ID_Counter will increment by one. Then the Data_Counters will reset and restart counting from zero to sample and update the next sensor data. In this protocol all counters in all nodes must be initialized to reset at the same time with the BS. All of these special counters in all sensor nodes and in the BS always follow their counting with the same rate by using unique clock generators in all nodes. This means they are all synchronized together based on their internal network time.

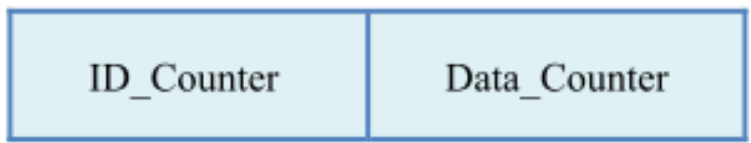

Fig 3: Counters. ID_Counter concatenated with Data_Counter.
Therefore, all Data_Counters in all nodes are counting together with the same initial value and with the same rate, and also it is the same for all ID_Counters in all nodes. Each sensor node has its own ID number as a constant value assigned to it permanently. According to the star topology defined for this work the sensor nodes will be scanned in order based on their ID number in a Cyclic-Broadcasting TDM technique as slaves by the BS as master node. Therefore sensor nodes will be selected for reading their data one at a time based on their ID, and each sensor node can recognize its time slot (Ti) by keeping the track of its ID_Counter to get activated, then reading its sensor data and sending it to BS. The sampling rate will be defined in network configuration as required for the applications. Sensor data in any sensor node is sensed by the sensor and detected by a sensitive low energy preamplifier. Then the sensor data which is an analog value as a real data will be forwarded to an ADC to convert to a binary value in desired number of bits based on the required resolution in the application [2-3].

\section{METHODOLOGY TO ENHANCE THE PERFORMANCE OF TBCD COMMUNICATION PROTOCOL}

TBCD communication protocol assumes that sensed parameters have a specific tolerance value. For instance, a sensor can read the blood glucose in range [3, 6.5] by tolerance of $0.5 \mathrm{mM}$. Therefore it can read a value out of these eight values; [3, 3.5, 4, 4.5, 5, 5.5, 6, and 6.5]. However, the tolerated threshold presents a serious problem especially for application which requires a high level of precision. Assigning a series of bits to each sensed value seems to be the perfect solution but it is still impractical to use since the size of the bit series may converge to infinite value. Our proposed method tends to resolve this issue using a distributed algorithm that assures a precise value of the physiological parameter with minimum energy consumption.Our method is based on defining a function $\mathrm{f}(1)$ which reduces a parameter value to a small number belonging to the interval [0, 11[ .A measured value can be included in one of the four Annulus as shown in Figure 4 :

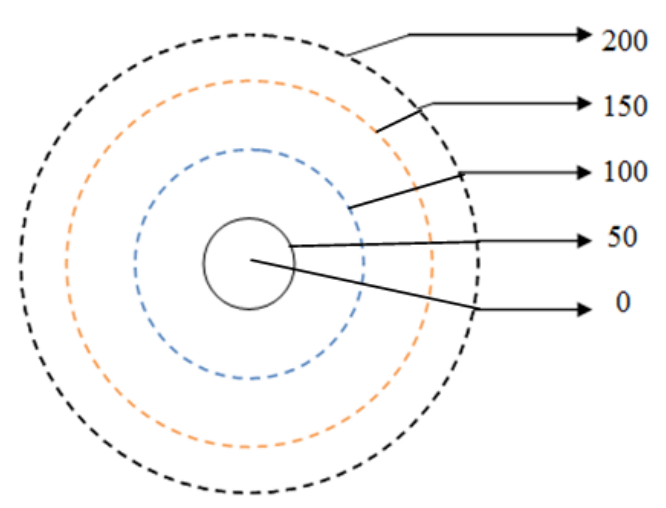

Fig 4: Classification of the measured parameters

$$
f(x, y)=(x-y) / 5 ;(1)
$$

$x$ and $y$ represents successively the measured value and the smaller radius of the Annulus.

Applying this function to such a number results in reducing it to a new number belonging to the interval $[0,11$ [ , which is 
going to be translated afterwards to a successive number of bits according to Table 1 , and for better accuracy, we extract three number of the decimal part .

Table 1. Data dictionary

\begin{tabular}{|c|c|}
\hline Binary representation & Decimal value \\
\hline 0 & 0 \\
\hline 1 & 1 \\
\hline 10 & 2 \\
\hline 11 & 3 \\
\hline 100 & 4 \\
\hline 101 & 5 \\
\hline 010 & 6 \\
\hline 011 & 7 \\
\hline 110 & 8 \\
\hline 111 & 9 \\
\hline 001 & 10 \\
\hline & \\
\hline
\end{tabular}

The structure of the transmitted data is depicted as follows:

\begin{tabular}{|l|l|l|l|l|l|}
\hline $\begin{array}{l}\text { Packet } \\
\text { Type } \\
\text { bit }\end{array}$ & $\begin{array}{l}\text { Data } \\
\text { Max 12 } \\
\text { bits }\end{array}$ & $\begin{array}{l}\text { Unit1 } \\
\text { 2bits }\end{array}$ & $\begin{array}{l}\text { Unit2 } \\
\text { 2bits }\end{array}$ & $\begin{array}{l}\text { Unit3 } \\
\text { 2bits }\end{array}$ & $\begin{array}{l}\text { Annulus } \\
\text { 2bits }\end{array}$ \\
\hline
\end{tabular}

Data bits: Random bit number of data.

Unit1: integer part's bit number.

Unit2: first number of the decimal part's bit number.

Unit3: second number of the decimal part's bit number.

Annulus Num: indicates which Annulus a number belongs to. The size of data packet is variable with a maximum value of 21 bits ( 9 control bits and 12 bits of data), and it depends on the number to convert. When data is received at the server side the value of data is then reconstructed using both the field depicted above in data fragments and the reduction function $\mathrm{f}$.

\section{EXPERIMENTAL RESULTS}

To evaluate the efficiency of our method, we wrote the simulation program with Java. The program begins with making a comparison of the energy consumed when the conversion function is used and when it's not so that to determine the type of data packet to send. As shown is Figure5 and Figure.6, our method minimize considerably the bit number of the measured parameter compared with its original binary representation and thus the transmission energy is minimized too. For instance instead of representing 4.98 value using 63 bits, the reduction function $\mathrm{f}$ minimizes its binary representation to 19 bits, therefore the energy consumption is decreasing from 3.17 to $0.95 \mathrm{~mW}$ [6].

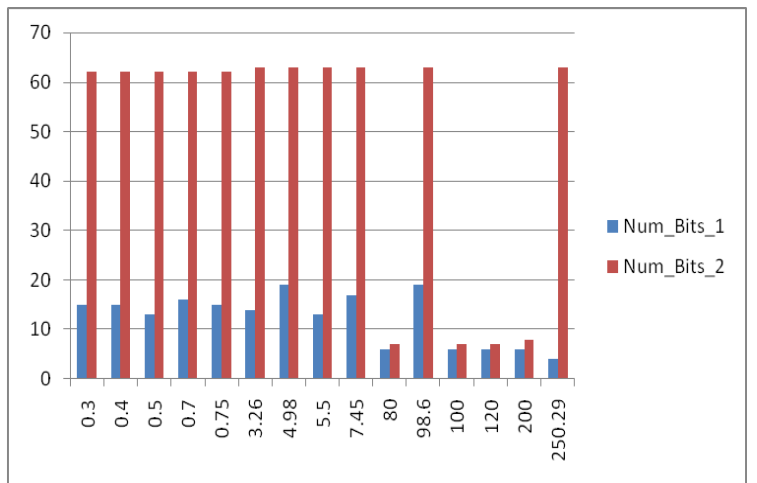

Fig 5: Bit number of a parameter value using the reduction function (Num_Bits_2) vs. using regular conversion (Num_Bits_1).

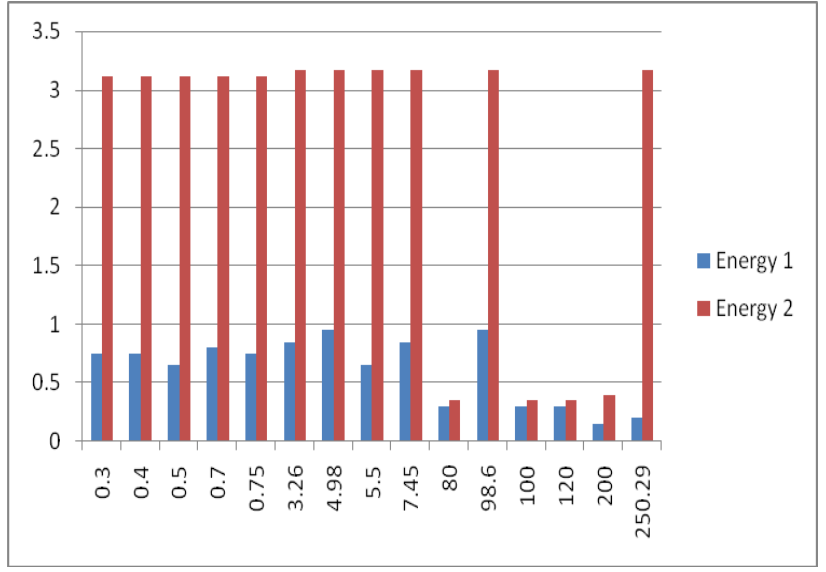

Fig 6: Energy of transmission using the reduction function (Energy 1) vs Energy of transmission using regular conversion of the parameter value (Energy 2).

\section{CONCLUSION}

Implanted sensor allows permanent monitoring of patient's information to the medical teams wirelessly through the world wide networks such as internet. In this paper, we introduced our contribution by proposing an enhanced version of TBCD communication protocol for minimum energy consumption and as a future work; we will be focusing on developing a new protocol to Minimize Thermal Effects of Implanted Biosensors which is based on multi-hop topology to reduce the high path loss around the human body.

\section{REFERENCES}

[1] Ashraf Darwish and Aboul Ella Hassanien.2011 Wearable and Implantable Wireless Sensor Network Solutions for Healthcare Monitoring. Sensors 11, 55615595; doi:10.3390/s110605561Ding,

[2] F., Fereydouni_Forouzandeh, O., Ait Mohamed, M Sawan.2008 Ultra Low Energy Communication Protocol for Implantable Body Sensor Networks. in Proc. IEEE NEWCAS-TAISA'08 Montreal pp. 57-60

[3] F, Fereydouni Forouzandeh, O., Ait Mohamed, M., Sawan, F. Awwad.2009 TBCD-TDM: Novel Ultra-Low Energy Protocol.

[4] Jamshid Abouei, David Brown, Konstantinos N. ,Plataniotis, Subbarayan Pasupathy. 2011 Energy Efficiency and Reliability in Wireless Biomedical Implant Systems. IEEE TRANSACTIONS ON INFORMATION TECHNOLOGY IN BIOMEDICINE.

[5] V., Shankar, A., Natarajan, S., K., S., Gupta L., Schwiebert. Energy-Efficient Protocols for Wireless Communication in Biosensor Networks. Dept of Computer Science and Engineering Computer Science Department Arizona State University Wayne State University Tempe, AZ 85287 Detroit, MI 48202.

[6] Xiaoling Wu, Brian J. d'Auriol, Jinsung Cho, Sungyoung Lee. 2008 Optimal Routing in Sensor Networks for Inhome Health Monitoring with Multi-factor Considerations.0-7695-3113-X/08 IEEE International conference on pervasive computing and communication.

[7] Yang, Guang-Zhong (Ed.). 2006 Body Sensor Networks. XXVIII, 36 pp. 
[8] Ren, H., \& Meng, M. Q. H. (2006) Rate control to reduce bioeffects in wireless biomedical sensor networks. In 3rd Annual international conference on mobile and ubiquitous systems Workshops, San Jose, CA, pp. 1-7.

[9] Tang, Q., Tummala, N., Gupta, S. K. S., \& Schwiebert, L.(2005) Communication scheduling to minimize thermal effects of implanted biosensor networks in homogeneous tissue. IEEE Transactions on Biomedical Engineering, 52(7), 1285-1294.

[10] Bag, A., \& Bassiouni, M. A. (2006) Energy efficient thermal aware routing algorithms for embedded biomedical sensor networks. In 2006 IEEE international conference on mobile adhoc and sensor systems (MASS), Vancouver, BC, pp. 604-609.

[11] Takahashi, D., Xiao, Y., Hu, F., Chen, J., \& Sun, Y. (2008) Temperature-aware routing for telemedicine applications in embedded biomedical sensor networks. EURASIP Journal on Wireless Communications and Networking, Vol. no. Article ID 572636, 11 p.

[12] D.Garrison."Minimizing Thermal Effects of In Vivo Body Sensors», Virginia Polytechnic Institute and State University, College of Engineering, Virginia, USA. 